\title{
Assessing the effectiveness of knowledge management in virtual manufacturing organizations
}

\author{
Ashok Kochhar and Craig Everitt \\ School of Engineering and Applied Science, Aston University, Aston Triangle, Birmingham \\ B4 7ET. United Kingdom
}

\begin{abstract}
Knowledge management within an individual organization is a complex process in itself. Difficulties with knowledge management are compounded when dealing with a number of partners in a virtual organization. Many important issues need to be considered. These include strategy, company employees, technology, security, use of knowledge, relevance and content of knowledge, knowledge flow, trust and relationship, needs and capabilities of knowledge system, external company knowledge and performance measures. Thus effective knowledge management in a virtual organization requires a strategic but relatively complex approach involving detailed consideration of a number of parameters associated with the issues listed above. Following an assessment of the issues, this paper outlines a framework for assessing the effectiveness of the knowledge management processes of partners in a virtual organization. The framework has been transformed into a detailed questionnaire for practical applications.
\end{abstract}

Keywords

Virtual Organisations, Knowledge Management.

\section{INTRODUCTION}

The environment in which organizations operate nowadays changes rapidly. It is characterised by shortened product life cycles, frequent technical changes and increasing competition especially with globalisation tendency. Increasingly companies have to concentrate on their core competencies. This focus on core competencies means that very few manufacturing organizations have all the capabilities, which will enable them to take timely advantage of the opportunities as they arise in the market. This has resulted in the creation of the so-called virtual enterprises.

A virtual enterprise can be regarded as a group of companies with different but complementary core competencies joined together to produce a certain range of products. So, where one company concentrates on design, another is in charge of manufacture and the marketing is left in the hands of a third partner. Extended supply chains involving suppliers at a number of levels are another important characteristic of a virtual organization. The partners in a virtual organization do not 
have to be located close to each other since all the information can be shared electronically. These partnerships can be disbanded once the goals that brought them together in the first place have been achieved and the further continuation of the virtual organization is of little value.

An equally important recent development is the recognition of knowledge as an important asset for the organization due to the economy shifting to a knowledge economy. In this environment knowledge is the key resource and critical to survival. In fact knowledge has become the major source of competitive advantage. Business organizations are realizing that to remain competitive they must explicitly manage their intellectual resources and capabilities. Knowledge management can be regarded as the process of creating an organizational knowledge base from which knowledge can be retrieved as and when need arises and used in new and complex situations. Effective knowledge management is dependent on the extent to which companies can create knowledge not in the head of an individual, but knowledge on which the company as a whole can act.

\section{DATA, INFORMATION AND KNOWLEDGE}

Data can be classified as discrete statements about reality. Information is organised, meaningful collections of data. Information and data exist in many forms around the world. No amount of technology can convert data and information into knowledge. Information can only become true knowledge through human interaction. Complex processes are needed for the understanding, contextualising and reconstructing of information to create knowledge. It is impossible to say how much knowledge a company possesses, as opposed to being able to determine how much data and information is available. Any organisation will typically have 3 types of knowledge [1].

1. Tacit knowledge typically refers to personal knowledge, which can be difficult to explain to others or document usefully. However, this sort of knowledge is used effectively for individual problem solving.

2. Explicit knowledge describes readily available policies and procedures, and other such information, that is easily documented, catagorised and distributed to employees. This can be regarded as "organizational memory".

3. Cultural knowledge is the common knowledge that dictates employees everyday behaviour and decision making, and is often linked with a company's overall philosophy.

Because knowledge, and the companies that possess it, differ greatly, it is hard to generalise about certain aspects of knowledge, such as its structure and representation. For knowledge management to work at all however, these aspects need to be considered carefully taking into account the specifics of the situation. Any amount of stored knowledge needs to categorised, so it can be identified when retrieval is necessary. A large amount of knowledge may need several layers of categorisation to be useful, especially in a multi-company environment.

It has already been established that data and information stored in documents and databases cannot be regarded as true knowledge. However, by capturing enough 
information concerning the knowledge being stored, such as its content and context, it can be easily reconstructed as knowledge when retrieved. However, knowing how much is 'enough information' can be a problem. Representing knowledge is not just concerned with explicitly expressing an employees knowledge in some medium. By indicating where knowledge can be found is an excellent way to ensure knowledge can be shared, without even attempting to decode the knowledge into an explicit form. This works well even for knowledge that would be hard to turn from implicit to explicit.

Stored knowledge is not static, and will need to be manipulated during its life. Knowledge may well need to be updated, combined or converted to be more productive or accessible for the future. In single organisations, this could involve a handful of like-minded employees. However, across multiple companies, it could cause problems. Knowledge cannot be used to its full potential when it is 'read-only'. Allowing all companies access to retrieve and edit knowledge is essential, and therefore some manipulation guidelines may be necessary to ensure the knowledge does not deteriorate while being manipulated by a number of individuals, in one or more companies, simultaneously.

\section{KNOWLEDGE MANAGEMENT IN VIRTUAL ORGANISATIONS}

The very nature of a virtual organisation requires its component companies to be super-efficient, and that in turn requires the virtual organisation 'company' to be better than anything the competition can accomplish [2]. Knowledge management can prove to be significant in achieving this, and to the success of the entire venture.

It has been said countless times that environments are changing, and that knowledge is becoming the one true resource of a company [3]. When companies come together, each company brings its core competencies; essentially their expertise and specialist knowledge. Without that knowledge, the virtual organisation would be unable to maintain itself as a functioning enterprise [4]. Without managing the knowledge suitably, the success of that enterprise is in jeopardy.

Like any resource that is relied upon, such as knowledge, it is natural a company should want to increase its scope and quality as quickly as possible. Entering into a virtual enterprise can dramatically enlarge and enhance the pool of knowledge available. However, the company, and the virtual enterprise, will need to ensure that all the knowledge is available and understood by all parties. The increase in knowledge available may also be overwhelming to the extent it is not utilised effectively or at all. Proper knowledge management can help a company manage the fluctuations of all aspects of the knowledge pool inherent with working with virtual organisations.

Knowledge being viewed as an intangible asset highlights the importance for its correct management. Comparatively, physical assets are easily measured, distributed and managed. An intangible asset will not remain valuable, especially not within a virtual organisation made up of many different companies, without attention. It is essential that knowledge management between companies ensures any knowledge is not devalued, rather trying to increase its value and usefulness instead. 
Technology is often consciously downplayed when discussing single organisation knowledge management. Technology becomes more important with knowledge management over a virtual organisation, as technology is often seen as the 'glue' that binds the component companies together. While this may be true, the technology is only important for communicating knowledge in whatever form is appropriate, and an effective knowledge management strategy will ensure technology is utilised successfully.

Virtual organisations are complicated and are extremely information intensive [5]. Virtual organisations are created to obtain the benefits of working closely with other companies, and much benefit is created from intense information presence, which in turn makes the virtual organisation complicated. Knowledge management can release the potential of the information by exploiting it for greater productivity, new value and increased competitiveness, while reducing the complexity of the situation [6].

The importance of knowledge management can be seen by the synergistic relationship between knowledge management and virtual organisations, and the development cycle of the concepts when combined [5]. A virtual organisation requires investment in knowledge management, and subsequent investments in knowledge management present new demands on the virtual organisation, and so the cycle continues.

There is the opportunity for many differences between members of a virtual organisation. However, knowledge management is an area that can provide some sort of coordination and integration with other members. The coordination of members with regards to knowledge is very important, especially if member activities are reliant upon each other and the knowledge used. A virtual organisation should appear as one organisation to its customers, and a well-integrated knowledge management initiative can help achieve this.

The benefits of knowledge management contribute to the virtual organisation being the 'best of the best', which is the foundation for the creation of a virtual organisation. Knowledge management has also brought much to virtual organisations that have increased their speed and competitiveness beyond on what was expected a decade ago. Whether in a virtual organisation environment, or a single company, ultimately knowledge itself is proving extremely important, and therefore its management is just as important.

\section{KNOWLEDGE MANAGEMENT ISSUES}

While knowledge sharing amongst a virtual organisation introduces several unique issues, many problems stem from original knowledge management problems, complicated further by the addition of the virtual network. The main issues are;

\section{Strategy}

A company's culture is arguably the most significant barrier against a successful knowledge management venture. There are many documented cases where the whole initiative has failed, despite significant investment in the technology and 
infrastructure. In a virtual organisation context, all parties should have a culture supportive of knowledge management, as there are at least two participants, and a successful transfer will need to be successful at both ends.

Knowledge sharing amongst a virtual organisation will need a slightly different culture supporting it. Employees will be learning to work and communicate differently in addition to sharing knowledge. The importance of the culture is such that it has been recommended that a successful venture starts by adapting the approach to fit in the current culture as best as possible [7].

Throughout the organisation as a whole there may be a fear of losing company stability or their market position [8]. It may be felt that the company practices and knowledge make the company unique, and to some extent this is true. The company needs to adapt its mindset in a similar fashion to the employees. A company should remind itself of the goals and benefits of knowledge sharing closely with virtual organisation partners. If it finds them not to be acceptable then the company has cause for concern. Sharing knowledge can highlight core concepts, not dilute them, leading to more business, not less [9]

With knowledge management, there is a lack of any concrete implementation frameworks. Most work within the field over the years has concentrated on 'what' to do, rather than 'how'. This potentially leaves a virtual organisation in a situation where multiple companies are trying to achieve the same thing via very different methods. This could cause problems when trying to share knowledge across the network, as they may be an incompatibility of procedures and understanding.

The key principle of virtual organisation is communication. Companies need to be able to communicate between themselves, usually over a distance. Practicing knowledge management over a virtual organisation requires the distant communication to carry more than usual. The communication links between companies will have to support certain knowledge processes, and the knowledge itself, without losing the effectiveness or meaning. While physically overcoming this may be a technical problem, how the technology is used, and how employees are to cooperate is very much an organisational issue.

To accomplish knowledge sharing between virtual organisation members will take a lot of company resources, much more than a regular internal project. A company must be able to access and commit the money, technology, skills and time. The issue of money is a simple one; the company can access the funding required and is willing to release it. Technology can be similarly acquired. The company must have employees who can implement and develop knowledge [9], which may prove difficult to attain. It is said the truly successful knowledge companies also have employees' actively seeking and creating knowledge [10]. This applies to all members of a virtual organisation.

A single company's attitude can be very important when working with other companies. For the relationship to operate at its potential, there should be no negative or dismissive attitudes. These attitudes can occur subconsciously, so a company must consciously check it is not behaving inappropriately. Within a virtual organisation, it is not likely all companies will be equal in terms of size. While this in itself is not bad, it can lead to the adoption of a domineering and controlling attitude towards smaller companies by larger members. This is common in company relationships, but not healthy. With knowledge management, a company believing 
itself to be more up-market than its associates, may be dismissive of companies it deems more down-market, and may take the view that its image, or knowledge, will be 'contaminated'.

A joint knowledge management venture should not be attempted unless at least one party has experience, with a well-established knowledge management programme already in place. This will lead to a much better chance of success. Other companies should ideally have some experience, although the more developed the other members, the more chance of conflicting practices, which may complicate the issue.

\section{Employees}

Many employees may be skeptical about knowledge sharing. This is common in any knowledge management initiative, whether it is within a single organisation, or across several. The first reaction an employee may have is that what they know is responsible for where they are today. "Knowledge is power" [11], so why give it up? There seems to be even less sense in sharing knowledge with other companies, which can create an internal resistance to sharing knowledge in order to protect company interests.

To combat these problems, employees' first need to see the rewards of sharing knowledge, the immediate benefits they themselves will experience, and the benefits to their company and other companies involved. By actively encouraging the sharing of knowledge, and employees feeling some benefit, their attitude should gradually change to incorporate knowledge management principles into their everyday routine.

Knowledge management can bring about conflict at five different levels: Country, Company, Hierarchy, Department and Individual [8]. These highlight the fact that knowledge management conflicts occur where there are significant differences between the parties sharing and receiving knowledge. Within a virtual organisation there is scope for the companies and employees sharing knowledge to be very different, and it is these differences that affect the creation of trust and their effectiveness of knowledge sharing.

Common differences include nationality, language, culture and legislature. These differences are not automatic problems, although they often become them, and when they do, companies will have to identify solutions and compromises to ensure optimum knowledge sharing.

Language can often be a problem, even if both parties speak the same language, although obviously different languages present more difficulties. Both scenarios can involve varying amounts of ambiguity in the sender's communication, and varying amounts of misunderstanding on the part of the receiver. For the knowledge sharing to be as successful as possible, both the ambiguity and misunderstanding should be minimised as much as possible. One strategy to achieve this is to define common terms and concepts.

These differences highlight the potential lack of any common ground or skills shared between virtual organisation partners. Shared contexts and common experiences are essential for effective communication [12], and with less than excellent communication, knowledge sharing also will suffer. 
Companies can try and create shared contexts and common experiences from which communication will benefit greatly; occasional face-to-face meetings for example would help enormously, as well as increase other important factors, such as the trust between parties and the trust in the knowledge being shared. Unfortunately, this may not be possible for all employees, or at all if many global companies are collaborating. Suitable substitutes could have a similar effect in these situations, for example video conferencing.

Even without meeting, employees from different firms can still build up a relationship. The level of the relationship can depend on the types of communication and the frequency of communication. The more familiar external colleagues become, the more effective their communication will be. The consequences for knowledge sharing are increased overall efficiency, including less lost knowledge, misunderstanding and wasted time.

The same concept can apply with the technology people use to share knowledge; increased familiarity can increase performance of both the technology and employee. The company can help boost familiarity and performance of employees with training in both using the knowledge technology, and the best practices and principles of sharing knowledge. The level of training relies on how important knowledge sharing is to the company, and how far the concept is to be advanced. As well as ensuring the company's employees are adequately trained, the competency of other virtual organisation members should be considered.

A company serious about knowledge needs a knowledge manager in some form or another. Other company resources are likely to have administrators, and knowledge will not become 'managed' without supervision [13]. In the case of a virtual organisation, knowledge managers will be able to discuss the development of any joint activities, coordinate efforts and reduce any incompatibility of knowledge systems as much as possible.

\section{Technology}

In practice a lot of emphasis is put on knowledge sharing technology, and often more important issues are not addressed correctly. While technology is important, and required for knowledge sharing, there are matters more vital for knowledge management success, such as the creation of a culture accepting knowledge sharing and exploitation [14]. The technology perhaps is more important however when combined with the virtual organisation context, as both concepts are dependant on it.

To implement knowledge management within a virtual organisation, the technology simply has to be there. Both models rely upon very similar technology for most functions, although certain areas may need specific solutions.

Technology supporting virtual organisations and collaborative work tends to concentrate on communication, often trying to negate such things as the distance or time between parties. Knowledge management technology focuses more on the ability to locate specific knowledge, although this doesn't always mean actually storing it electronically. Combining both concepts leads to the need for more advanced abilities, such as the capability to request and receive knowledge over distance and time. 
Generic, pre-designed solutions do exist for managing knowledge and working within a virtual network, although their validity should come under question. Preexisting solutions may however have certain benefits, such as lower costs. Companies situations and needs will vary, and most certainly different companies will want to manage different types of knowledge, so despite their possible benefits, generic solutions may not fulfil a company's, or group of companies, real requirements.

One issue that is undoubtedly present in most projects involving technology is that of compatibility. In a virtual organisation context, it is not just inter-department compatibility, but inter-organisational compatibility. With the wide range of possible scenarios and solutions, it is unlikely that two companies will have the same set-up. This is not necessarily a problem, as there are varying levels of compatibility, or measures to create compatibility. The importance of the compatibility issue ultimately depends on how ambitious the level of interaction will be between companies.

\section{Security}

Inevitably an organisation will not want to share all of its knowledge, even with close working partners. Knowledge security should be an important aspect of any knowledge initiative, with there being many different related issues. Primarily, an organisation should protect the overall security of sensitive material from all parties. Knowledge that is shared with external bodies should be accessible to only those intended, which can be a serious issue if an open technology such as the Internet is used. There is also a possibility that shared information may be intentionally, or unintentionally, exposed to the competition. This could lead to internal resistance, with employees aspiring to safeguard the interests of the company by not fully sharing company knowledge with external parties. There may be fears that knowledge will filter through suppliers/customers eventually improving the competition. With regards to the virtual organisation, an organisation should outline at every level what knowledge should be shared, and know what knowledge is shared, and how.

\section{Company use of knowledge}

When working closely with other companies, employees will hopefully find a healthy flow of knowledge sent and received by the organisation. This presents the problem of what to do when external knowledge updates current company thinking, which can potentially affect any virtual organisation member. An average employee may reject the knowledge, or not adapt to it quickly. However this may be very counter productive, as any advantage the knowledge yielded will not benefit the company while the knowledge is not fully taken on board. The organisation should ensure that it is not slow reacting to new knowledge presented to it [15].

External knowledge may disagree with internal knowledge, as opposed to updating it. These situations of knowledge conflict, where both items of knowledge cannot be correct, must be dealt with effectively otherwise they may cause further problems. The incorrect knowledge must be discarded, and it must be ensured that 
correct knowledge is used in the organisation. It may be impractical to verify all conflicting knowledge to resolve the problem, so other things may be taken into account, such as the knowledge source.

External knowledge should be verified occasionally, just as internal knowledge should be. It is all part of the knowledge management process to ensure the knowledge used by the company is of high enough quality, and knowledge from the virtual network should be no different. Verifying external knowledge does not mean the knowledge is not trusted, that is itself a separate issue. Knowledge verification can in fact help build up trust if appropriate.

A company should ensure it does not become too dependent on its partners for knowledge. If a company relies on a lot of external knowledge for its products or services, it leaves itself open to opportunistic behaviour, or being tied to one partner unnecessarily. The company may want to ask itself why the knowledge cannot be created internally if it is such a necessity to their production.

\section{Relevance and content of knowledge}

"Information needs to be targeted if it is to serve knowledge." [16] It should be known what purpose the knowledge may provide, and perhaps more importantly, who might access it. This is true for a single organisation, yet even more so for multiple companies within a virtual organisation. With respect to knowledge, the company must be aware it is not working alone. If some thought is given about the intended receiver when expressing or communicating knowledge, this can help increase the understanding and decrease time wasted.

A company may have several products or services, but only collaborate within a virtual organisation for a few from their portfolio. The remaining concerns may be associated with their own knowledge items, which may be made available to the virtual organisation through a common gateway. While the knowledge being available is not a security concern, the knowledge available to the virtual organisation should be restricted to what is necessary. Decreasing the amount of knowledge accessible may increase the overall effectiveness of communication, by reducing factors such as information overload.

However companies arrange their knowledge to be available across the virtual organisation, it is important that all companies can access the same up to date knowledge. A virtual organisation may depend on taking as much advantage of knowledge as possible, but if some partners access outdated knowledge, then the advantage is gone, or the knowledge becomes worthless. By synchronising the knowledge, the knowledge management can become as effective and useful as within a single organisation.

\section{Knowledge flow}

An important point to make is that any knowledge management technology should be capable of storing and transferring knowledge in a variety of ways. This is to accommodate the different types of knowledge, and the varying complexities of that knowledge adequately, without any knowledge being lost. This is especially true when the communication is across a virtual organisation. The communicating 
employees may have little experience with the knowledge or each other, so the technology must be able to make the process as efficient and easy as possible.

Successful knowledge transfers between companies can be described theoretically, and a company must ensure the description holds true for its own interorganisational knowledge sharing. The knowledge being shared should not become devalued because of its reception by another company. The knowledge being received should be complete and become part of the receiver's knowledge stock [15].

The knowledge should ideally flow in both directions, and often. While this can indicate a successful knowledge sharing system, it can present a problem to the company, as it may want to monitor its knowledge transactions. A company should know what knowledge is available to external companies, and from where. As employees become more familiar with the concept and their colleagues, knowledge sharing may take on more and more personal and informal guises. Companies may be interested in how successful these interactions are, but will also be concerned over any possible security breaches.

\section{Trust \& Relationship}

Trust is an important concept in both knowledge management and the virtual organisation fields. There are two main trust issues for employees. Employees may not trust the people who want to use their knowledge, or trust the knowledge they receive. Internally this poses less of a problem, as there is more scope for trust having already been built up, or to be formed quickly. With employees and their external colleagues, the formation of trust may be very difficult and slow; there are many extra factors that may hinder the development of trust. Such factors may also prove to be barriers to effective inter-organisational knowledge sharing.

Trust has long been identified as important in business-to-business relationships, although strategies for creating and measuring it are lacking [17]. Trust can be thought of as a situation where one party has the opportunity to disadvantage another, but it is not a realistic probability. The trust issues a company may experience are similar to the issues an employee faces in the situation of sharing knowledge within a virtual organisation. A company will first have to decide to trust a company, and then the knowledge it subsequently provides. For successful operations, there will have to be high levels of trust within the virtual organisation, whether it is justified or not.

Unfortunately, trust cannot be strictly enforced; it must be forged, usually over a period of time, although the phenomenon of high initial trust values leading to successful relationships has been observed. Some aspects of company relationships do have to be formal, especially legal issues. The higher the levels of trust, the more features a relationship can include, and this should be considered. A company must ensure enough trust has built up before commencing advanced initiatives that will fail without higher levels of trust. While trusting a company is not an exact science, and sometimes companies will needed to be trusted with no solid foundation, as soon as a company has acted in a fashion deemed untrustworthy, relationships should be examined, exercised with caution, or terminated at the company's discretion. 


\section{Needs and ability of knowledge system}

An important issue for the knowledge system, from an employee perspective, is being able to be credited for knowledge contributions. Lack of ability to determine whom to credit as contributing the knowledge can reduce the quality of knowledge held, and the amount of knowledge shared. An employee may not want to share what they believe to be valuable knowledge if anyone can use it to their advantage. Conversely, poor quality knowledge may be submitted if knowledge sources are essentially anonymous. The issue may be more significant with multiple companies, all wanting recognition for their knowledge input. An identifiable source can also help knowledge receiver put more trust in the knowledge.

One process of knowledge management is the advancing or refining of current knowledge through such means as group discussion. This requires a very high level of communication in terms of efficiency and communication capacity. The first choice would be face-to-face interaction, however this may not be possible on a regular basis, or at all with, virtual partners. Nonaka [18], among others, cite face-toface communication as being unsurpassed. It could be argued that since this was said over a decade ago, more sophisticated communication technology, which can approximately replace such a high level of communication, is more available and feasible. Whatever the conclusion, an organisation must ensure all areas of knowledge management are followed, not just the processes that are the easiest to accomplish across a virtual network.

\section{Extermal company knowledge}

A company should understand what knowledge virtual organisation members hold. A company may implicitly presume all parties hold the same assumptions and tacit knowledge, and codify their own knowledge accordingly. This could leave the knowledge useless to anyone outside the organisation, or make communicating it an intensive process. A company should not assume what other companies know, and perhaps formalise what is regarded internally as 'common knowledge' for partners. Companies should also make it clear what knowledge they possess and are willing to share. This ensures any company within a virtual organisation can find the knowledge required, and knows what is needed to communicate knowledge to others.

A company will interact with many different companies, with varying amounts of contact, which can generate a lot of knowledge. This knowledge will be lost if it is not consciously made explicit. Some of the knowledge may not seem important, but can provide a useful insight in the future when decisions need to be made. Being able to consult the companies knowledge will also help build on any former relationships or trust previously formed that may not have been recognised, especially if previously other employees or departments were responsible for the contact.

Companies should not only share knowledge with their partners, but compile knowledge about them as well. This knowledge should be kept separate from their subject. The company should know certain details about their partners, which can be used to appraise them, and help them where there is mutual benefit. An example 
would be to know what level of knowledge training their employees receive. It is in the interest of the company to ensure its partners are competent in sharing knowledge. Knowledge about partners' competitors should also be considered, as they can be used to appraise partners in terms of their market. Differences between companies can cause problems with communication and knowledge sharing, and a company should know what obvious, and not so obvious, differences there are between members.

\section{Performance measures}

Companies will be keen to measure the performance of knowledge management internally and its performance across the virtual organisation. There are no 'standard' tests that can show confidently how knowledge management is positively affecting processes using it. Any effective performance measures would also need to be more intensive than simply collecting quantitative data, and would need to be more intrusive into certain processes. This may make it more difficult, as virtual organisation members may not want to commit the time required, or want to share that sort of process information.

Practicing knowledge management within a virtual organisation is not an immediate achievement, and a project will go through many stages before becoming an important part of an organisation. An organisation will want to measure its interorganisational knowledge efforts at every stage, but must realise that at different times in the knowledge management lifecycle, different measures should be used, and some measures, while calculable, are not always useful or valid at that particular time [2].

\section{Assessment of Effectiveness of Knowledge Management in Virtual Organisations}

To help manufacturing organisations assess the effectiveness of their knowledge management processes, especially as they become part of supply chains and virtual organisations, a detailed diagnostic system has been created, which takes account of the issues highlighted in the previous section. As part of the development process a considerable amount of work was carried out with a number of organisations, at different stages of knowledge management, to ensure that it reflects the realities on the ground. The diagnostic consists of a total of 96 questions, split as follows between different issues identified above.

$\begin{array}{lcc}\text { Strategy } & - & 13 \text { questions } \\ \text { Employees } & - & 12 \text { questions } \\ \text { Technology } & - & 6 \text { questions } \\ \text { Security } & - & 3 \text { questions } \\ \text { Company Use of knowledge } & - & 6 \text { questions } \\ \text { Relevance and content of knowledge } & - & 8 \text { questions } \\ \text { Knowledge flow } & - & 9 \text { questions } \\ \text { Trust and relationship } & - & 6 \text { questions } \\ \text { Needs and capability of system } & - & 8 \text { questions } \\ \text { External company system } & - & 9 \text { questions }\end{array}$


It is currently being implemented as part of a Web based system to allow organisations to assess their current situation, i.e. the strengths and weaknesses of their knowledge management system. The answers to the questions can take a number of different forms, for example Yes/No, multiple-choice, or on a scale. Questions also incorporate an explanation to help a user fully understand a question before answering it. The answers given to individual questions are assessed against the desirable set of conditions for effective knowledge management in virtual organisations. Formal feedback is provided in relation to each individual question, including the actions, which should be taken to overcome the weaknesses. The final report based on all the questions priortises the actions into the short-term actions, which can be implemented quickly and at low cost, and will provide immediate benefits, and the long term actions requiring significant change and investment.

\section{CONCLUSIONS}

This paper has outlined the main issues associated with knowledge management as they affect organisations operating as part of a virtual enterprise. Work with organisations at different stages of knowledge management has demonstrated that many of these issues are very different from the knowledge management issues within an organisation, which is not part of a virtual enterprise. A diagnostic system has been developed to help organisations identify their strengths and weaknesses, as well as the actions necessary to overcome the identified weaknesses.

\section{ACKNOWLEDGEMENT}

The authors are grateful to the Engineering and Physical Sciences Research Council (EPSRC) for the award of a research grant to undertake the work described in this paper.

\section{REFERENCES}

[1] CW Choo, The Knowing Organization: How Organizations Use Information to Construct Meaning, Create Knowledge, and Make Decisions, Oxford University Press, New York, 1998.

[2] Jonathan Franks, The Virtual Organisation, Work Study, 1998, Vol. 47(4), 130 - 134.

[3] Yogesh Malhotra, TOOLS@WORK: Deciphering the Knowledge Management Hype, Journal for Quality \& Participation, 1998, Vol. 21(4), 58 - 60.

[4] Thomas H. Davenport \& Laurence Prusak, Working Knowledge: How Organizations Manage What They Know, Harvard Business School Press, 1998.

[5] David G. Schwartz, Internet-Based Organizational Memory and Knowledge Management, Idea Group Publishing, 2000.

[6] John Davies, Knowledge Management: the Tools for the Business, BT Technology Journal, http: //www.sc-server1.bt.com/bttj/, 1999, Vol. 18(1). 
[7] Richard McDermott \& Carla O'Dell, Overcoming cultural barriers to sharing knowledge, Journal of Knowledge Management, 2001, Vol. 5(1), 76 - 85.

[8] Kulwant Pawar et al, Inter-Organisational Knowledge Management: Focus on Human Barriers in the Telecommunications Industry, CE2001 - Proceedings of the 8th ISPE International Conference on Concurrent Engineering, California, USA, 2001.

[9] Richard J Barson et al, CORMA - Inter- and Intra-Organisational Barriers to Sharing Knowledge in the Extended Supply chain, In: E-business - Key Issues, Applications, Technologies, Stanford-Smith, 2000, 367 - 373.

[10] P. Trott, Inward technology transfer as an interactive process, Technovation, 1995, Vol. $15(1)$.

[11] Stavros Kammas, Knowledge Management in Virtual Environments, INFORMATION TECHNOLOGIES AND KNOWLEDGE CONSTRUCTION: Bringing Together the Best of Two Worlds, 5th Human Centred Technology Postgraduate Workshop, 2001.

[12] Tina Brandt Husman, Efficiency in Inter-Organisational Learning: A Taxonomy of Knowledge Transfer Costs, Copenhagen Business School, Department of Industrial Economics \& Strategy, Working Papers 01 - 4, 2001.

[13] Thomas H. Davenport, Principles of Knowledge Management, Graduate School of Business, University of Texas at Austin, 1996, http: //www.bus.utexas.edu/kman.

[14] Helen Gumbley, Knowledge management, Work Study, 1998, Vol. 47(5), 175 - 177.

[15] Cynthia Hartz et al, Measurement for Knowledge Management, American Productivity \& Quality Centre, 2001, http: //www.apqc.org/free/articles.

[16] H Scarbrough, J Swan \& J Preston, Knowledge Management: A Literature Review, People Management Series, Institute of Personnel Development, London, 1999.

[17] Keith Blois, A trust interpretation of business-to-business relationships: a case-based discussion, Management Decision, 1998, Vol. 36(5), 302 - 308.

[18] Ikujiro Nonaka, The Knowledge-Creating Company, Oxford University Press, 1995. 